05,01

\title{
Магнитокристаллическая анизотропия спеченных магнитов (PrDy)(FeCo)B
}

\author{
() Е.И. Куницына ${ }^{1}$, В.П. Пискорский ${ }^{2}$, Д.В. Королев ${ }^{2}$, Р.А. Валеев ${ }^{2}$, \\ B.В. Кучеряев ${ }^{2}$, Р.Б. Моргунов ${ }^{1,2, \text { Ф }}$ \\ ${ }^{1}$ Институт проблем химической фризики РАН, \\ Черноголовка, Россия \\ ${ }^{2}$ Всероссийский институт авиационных материалов, \\ Москва, Россия \\ ๆ E-mail: morgunov2005@yandex.ru
}

(Поступила в Редакцию 10 мая 2018 г.)

Магнитокристаллическая анизотропия одноосных редкоземельных магнитов (PrDy)(FeCo)B определена с помощью модели Акулова и исследована в диапазоне температур $2-300 \mathrm{~K}$. В образцах $(\mathrm{PrDy})(\mathrm{FeCo}) \mathrm{B}$ наблюдается степенная зависимость константы анизотропии $K(T)$ от намагниченности насыщения $M_{S}(T)$ в диапазоне температур $2-300 \mathrm{~K}$ с показателями степени $m=1.7-2.1$ в соответствии с эмпирическим правилом Callen-Callen. B образцах с примесью $\mathrm{Gd}$ обнаружено отклонение от степенной зависимости $K\left(M_{S}^{m}\right)$. Обсуждаются возможные причины отклонения от правила Callen-Callen и вариации показателя степени $m$.

DOI: $10.21883 /$ FTT.2018.12.46728.128

\section{1. Введение}

Главный фактор, управляющий температурной и временной стабильностью постоянных магнитов, - магнитокристаллическая анизотропия, создаваемая редкоземельной подрешеткой в магнитах семейства RE-TM-B и RE-TM. Существующие теоретические представления о температурной зависимости константы магнитной анизотропии $K$ и ее связи с химическим составом сплава лишь отчасти соответствуют экспериментальным данным [1]. Интерес к этой проблеме обусловлен не только необходимостью развития фундаментальных представлений о магнетизме, но и поиском условий, необходимых для создания температурно- независимых магнитных свойств постоянных магнитов, необходимых во многих устройствах. Эта практическая задача сложна и может быть решена лишь в узком диапазоне температур вблизи точки эксплуатации магнитов, поскольку магнитокристаллическая анизотропия в большинстве случаев сильно зависит от температуры [2-4]. Теоретическое описание температурной зависимости магнитной анизотропии в редкоземельных магнитах является нетривиальным и часто спорным [5-8]. В частности, в литературе обсуждается применимость теории Callen-Callen, предсказывающей степенную зависимость константы анизотропии от намагниченности насыщения $K \sim M_{S}^{m}$, где $m=3$. В рамках классической модели Гейзенберга, где нормированный классический спин взаимодействует с самосогласованным средним полем, при низких температурах ожидается выполнение теории Callen-Callen в редкоземельных магнитах [9]. Для одноподрешеточных магнитов, например Fe и Co, теория Callen-Callen точно описывает экспериментальные данные. Однако уже для сплавов $L 1_{0}$ (FePt, FePd, FeNi, CoPt и CoPd), где температурная зависимость намагниченности и температурная зависимость анизотропии имеют разные причины, это правило не выполняется $(m=2.1)[8,9]$. В [8] отмечается, что отклонение от теории Callen-Callen связанно с эффективным анизотропным обменом с атомами $\mathrm{Pt}$ и предполагается, что этот механизм является общей чертой сплавов $3 d-5 d$ и $3 d-4 d$ с относительно сильной спин-орбитальной связью и чувствительностью магнитной анизотропии к коэффициентам Стонера.

В [6] было установлено, что для сплавов PtCo, PdFe и $\mathrm{PtFe}$ температурное поведение анизотропии можно описать с помощью двухподрешеточной модели (в отличие от одноподрешеточной модели в теории Callen-Callen). Авторы [10] получили линейную зависимость магнитной анизотропии от намагниченности насыщения со значением $m=1.9-2.1$ для сплавов $\mathrm{FePt}, \mathrm{CoPt}, \mathrm{MnAl}$, FeCo. Закон Callen-Callen может также нарушаться в случае, когда имеются атомы с сильной спин-орбитальной связью, которые вносят вклад в анизотропию, но не оказывают заметного влияния на другие магнитные свойства $[6,8,11,12]$.

Описанные выше случаи нарушают три основных предположения теории Callen-Callen: одиночные ионы с локализованными магнитными моментами рассматриваются как основная причина намагниченности материала; спин-орбитальное взаимодействие рассматривается как небольшое возмущение обменной связи в материале; и наконец, температурная зависимость намагниченности и анизотропии имеет одно и то же происхождение. Применения теории Callen-Callen для описания магнитной анизотропии монокристаллов $\mathrm{Nd}_{2} \mathrm{Fe}_{14} \mathrm{~B}$ не часто встречаются в литературе. Теоретические работы $[13,14]$ предсказывают выполнение теории Callen-Callen с показателем степени $m \approx 2$ в этих кристаллах. Вместо монокристаллов $\mathrm{Nd}_{2} \mathrm{Fe}_{14} \mathrm{~B}$ в технике используют спе- 
Состав исследуемых образцов, способ приготовления и соответствующая температура прессования, а также значение показателя степени $m$ в законе Callen-Callen. (Прочерки означают отсутствие степенной зависимости между константой анизотропии и намагниченностью насыщения при разных температурах)

\begin{tabular}{|c|c|c|c|c|}
\hline $\begin{array}{c}\text { № } \\
\text { образца }\end{array}$ & Состав & $\begin{array}{c}\text { Способ } \\
\text { приготовления }\end{array}$ & $\begin{array}{c}\text { Температура } \\
\text { деформации, }{ }^{\circ} \mathrm{C}\end{array}$ & $m$ \\
\hline 1.1 & \multirow{3}{*}{$\begin{array}{c}\left(\operatorname{Pr}_{0.53} \mathrm{Dy}_{0.47}\right)_{13.43} \\
\left(\mathrm{Fe}_{0.64} \mathrm{Co}_{0.36}\right)_{79.06} \mathrm{~B}_{7.51}\end{array}$} & \multirow{2}{*}{ экструзия } & 900 & 1.8 \\
\hline 1.2 & & & 850 & 2.1 \\
\hline 1.3 & & $\begin{array}{c}\text { прямая } \\
\text { деформация }\end{array}$ & 850 & 2.0 \\
\hline 2.1 & \multirow{3}{*}{$\begin{array}{c}\left(\operatorname{Pr}_{0.54} \mathrm{Dy}_{0.47}\right)_{13.26} \\
\left(\mathrm{Fe}_{0.64} \mathrm{Co}_{0.36}\right)_{77.61} \mathrm{~B}_{9.13}\end{array}$} & \multirow{2}{*}{ экструзия } & 900 & 1.8 \\
\hline 2.2 & & & 850 & 1.7 \\
\hline 2.3 & & $\begin{array}{c}\text { прямая } \\
\text { деформация }\end{array}$ & 850 & 2.1 \\
\hline 3.1 & \multirow{3}{*}{$\begin{array}{c}\left(\mathrm{Pr}_{0.51} \mathrm{Dy}_{0.36} \mathrm{Gd}_{0.13}\right)_{12.82} \\
\left(\mathrm{Fe}_{0.64} \mathrm{Co}_{0.36}\right)_{80.36} \mathrm{~B}_{6.82}\end{array}$} & \multirow{2}{*}{ экструзия } & 900 & - \\
\hline 3.2 & & & 850 & - \\
\hline 3.3 & & $\begin{array}{c}\text { прямая } \\
\text { деформация }\end{array}$ & 850 & - \\
\hline
\end{tabular}

ченные магниты $(\mathrm{PrDy})(\mathrm{FeCo}) \mathrm{B}$, для описания которых невозможно a priori использовать данные, полученные ранее для $\mathrm{Nd}_{2} \mathrm{Fe}_{14} \mathrm{~B}$. Вместе с тем известно, что семейство магнитов $(\mathrm{PrDy})(\mathrm{FeCo}) \mathrm{B}$ сильно отличается от $\mathrm{Nd}_{2} \mathrm{Fe}_{14} \mathrm{~B}$ относительно широкими температурными участками постоянной намагниченности (температурной стабильностью) и, следовательно, обладает определенной спецификой температурного поведения магнитной анизотропии. По этой причине магниты $(\mathrm{PrDy})(\mathrm{FeCo}) \mathrm{B}$ называют супермагнитами 4-го поколения [15-17]. Поэтому в данной статье предметом исследования была магнитная анизотропия в группе сплавов $\mathrm{RE}-\mathrm{FeCo}-\mathrm{B}$ ( $\mathrm{RE}$ - ионы редкоземельных металлов $\mathrm{Pr}, \mathrm{Dy}, \mathrm{Gd}$ ). Основная магнитная фаза 2-14-1 этих сплавов, как и $\mathrm{Nd}_{2} \mathrm{Fe}_{14} \mathrm{~B}$, имеет тетрагональную кристаллическую структуру. Хотя группа сплавов $\mathrm{RE}-\mathrm{FeCo}-\mathrm{B}$ сходна по строению и свойствам с хорошо изученной системой $\mathrm{NdFeB}$, это не дает оснований считать закономерности температурного поведения анизотропии одинаковыми. Еще одна особенность данной работы заключается в том, что в ней используются спеченные материалы. В спеченных магнитах на границах зерен существует поверхностная анизотропия с иной зависимостью от температуры [18-19]. Для монокристаллов $\mathrm{Nd}_{2} \mathrm{Fe}_{14} \mathrm{~B}$ в работе [1] экспериментально показано, что $K$ линейно зависит от $M_{S}(T)$ (т.е. по теории Callen-Callen показатель степени $m=1)$. В литературе не удается найти систематические исследования применимости теории Callen-Callen для многих других редкоземельных магнитов. Исследование новой группы редкоземельных магнитов в данной работе может восполнить этот пробел и приблизиться к получению общих представлений о свойствах магнитной анизотропии в них. Поэтому целью нашей работы было установление применимости теории Callen-Callen для описания температурной зависимости магнитной анизотропии в спеченных магнитах $(\mathrm{PrDy})(\mathrm{FeCo}) \mathrm{B}$ и $(\mathrm{GdPrDy})(\mathrm{FeCo}) \mathrm{B}$, приготовленных различными способами.

\section{2. Методика экспериментов и приготовление образцов}

Химические составы исследованых образцов $(\mathrm{PrDy})(\mathrm{FeCo}) \mathrm{B}$ представлены в таблице. Образцы изготавливались методом горячего компактирования и последующей горячей деформации компактов быстро закаленного порошка со средним размером микрочастиц до $200 \mu \mathrm{m}$. Порошки всех составов были получены при одинаковой линейной скорости вращения диска-охладителя установки „Кристалл-702“. Компактирование и деформация проводились на установке искрового плазменного спекания в графитовых пресс-формах. Полученные компакты были разделены на три серии. Первая и вторая серии подвергались горячей обратной экструзии при температурах $900^{\circ} \mathrm{C}$ и $850^{\circ} \mathrm{C}$ соответсвенно. Третья серия компактов подвергалась горячей прямой деформации при температуре $850^{\circ} \mathrm{C}$. Магнитная текстура в образцах, полученных методом прямой деформации, формируется параллельно оси приложенного давления, в то время, как в образцах, полученных экструзией, макнитная текстура перпендикулярна направлению оси прессования. (Под магнитной текстурой понимается преимущественное направление осей главной намагниченности микрочастиц основной магнитной фазы, из которых сформирован спеченный магнит). Полевые зависимости магнитного момента образцов в магнитном поле до $50 \mathrm{kOe}$ и 
при температурах 2-300 K были получены с помощью СКВИД магнетометра MPMS 5XL Quantum design.

\section{3. Экспериментальные результаты}

Для трех серий образцов (см. таблицу) были получены петли магнитного гистерезиса при температурах от 2 до $300 \mathrm{~K}$ с целью определения намагниченности насыщения $M_{S}$ и извлечения количественной информации о магнитной анизотропии (рис. $1, a)$. Константы магнитной анизотропии первого и второго порядков $K_{1}$, $K_{2}$ могут быть определены из анализа полевых зависимостей $M(H)$ в ,легкой ориентации“ с помощью формулы Акулова для кристаллов с одной легкой осью намагничивания [20]. Для этого были аппроксимированы высокополевые части петель магнитного гистерезиса $\left(H>H_{C}\right)$ были аппроксимированы выражением [20]

$M(H)=M_{S}\left(1-\frac{4 K_{1}^{2}}{15 M_{S}^{2} H^{2}}-\frac{64 K_{1} K_{2}}{105 M_{S}^{2} H^{2}}-\frac{128 K_{2}^{2}}{315 M_{S}^{2} H^{2}}\right)+\chi H$,

где $\chi H-$ слагаемое, описывающие вклад парамагнитной примесной фазы. Пример аппроксимации показан на pис. $1, b$. Процедура аппроксимации была проведена для всех трех серий образцов в диапазоне температур от 2 до $300 \mathrm{~K}$. На рис. 1, с приведена зависимость константы анизотропии первого порядка $K_{1}$ от температуры для образца 2.1.

Закон Callen-Callen был получен в предположении однородных ферромагнитных и одноионных анизотропных систем при температурах, далеких от температуры Кюри $\left(T \ll T_{C}\right)[21]$

$$
\frac{K(T)}{K(0)}=\left(\frac{M_{S}(T)}{M_{S}(0)}\right)^{m}, \quad m=\frac{l(l+1)}{2},
$$

где $K(0)-$ константа анизотропии при наименьшей доступной нам температуре $2 \mathrm{~K}, M_{S}(0)-$ намагниченность насыщения при температуре $2 \mathrm{~K}, m-$ показатель, зависящий от симметрии кристалла и степени корреляции между направлениями смежных спинов. $l-$ порядок сферической гармоники электронной волновой функции, определяющей угловую зависимость локальной анизотропии, при этом $l / 2$ равно порядку константы анизотропии. Здесь и далее принимаем, что эффективная константа анизотропии $K(T) \approx K_{1}(T)$.

На рис. 2, а приведены примеры зависимостей приведенной анизотропии $K(T) / K(0)$ от приведенной намагниченности насыщения $M_{S}(T) / M_{S}(0)$ в логарифмическом масштабе. Спрямление этих зависимостей в двойных логарифмических координатах означает, что константа анизотропии $K$ прямо пропорциональна $\left(M_{S}\right)^{m}$ во всем диапазоне температур. При этом значения показатели $\mathrm{m}$ варьируются в широком диапазоне от 1.7 до 2.1 в зависимости от способа изготовления образцов (см. таблицу). Соответствующие значения $l$ лежат в диапазоне $l=1.4-1.6$ (см. таблицу). Это соответствует примешиванию состояний с $l=1$ и $l=2$ в примерно одинаковой пропорции, что характерно для Dу при комнатной температуре. Для образцов серии 3 с добавкой
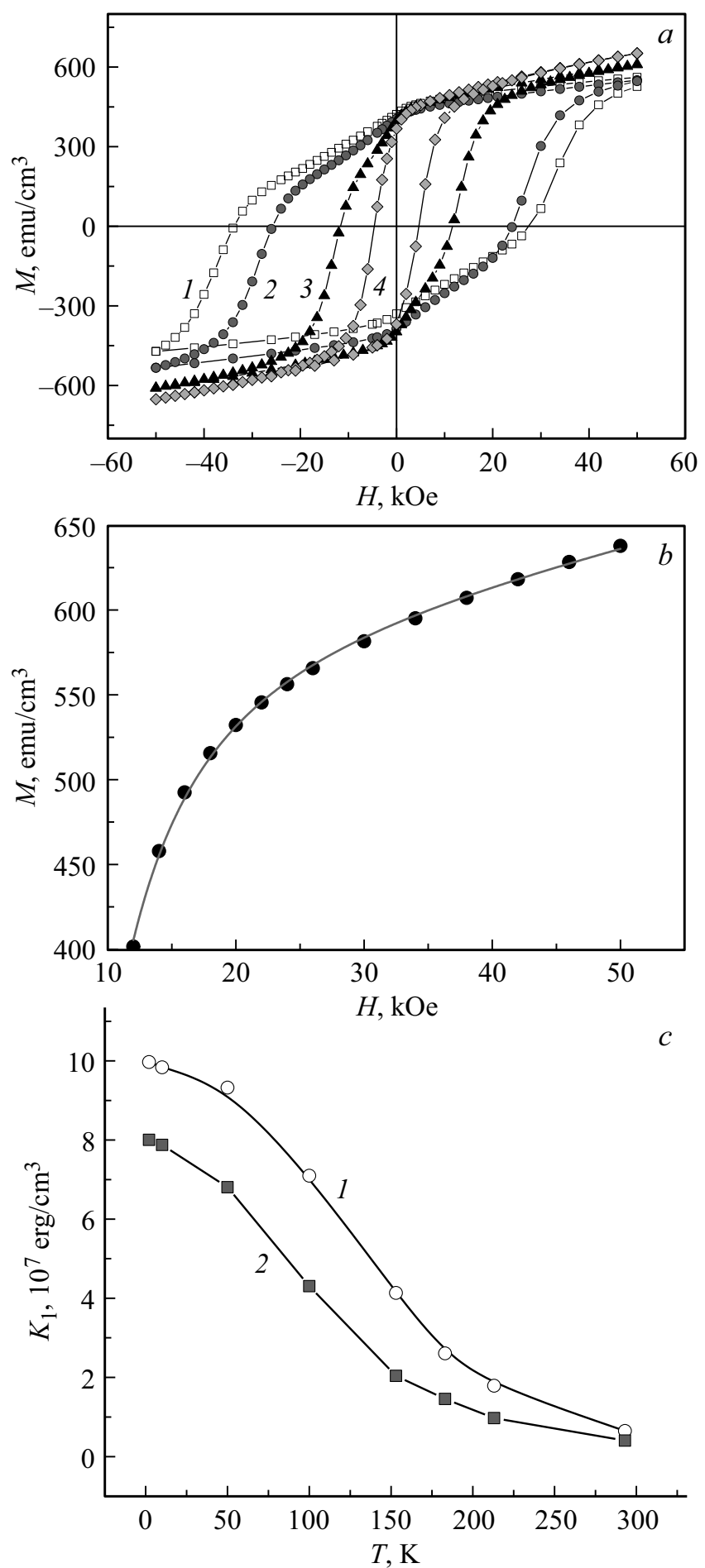

Рис. 1. $a-$ зависимость намагниченности образца 2.1 от поля при температурах: $2 \mathrm{~K}-(1), 100 \mathrm{~K}-(2), 210 \mathrm{~K}-(3)$, $300 \mathrm{~K}-(4) ; b-$ зависимость намагниченности образца 2.1 от магнитного поля. Сплошной серой линией показана аппроксимация зависимости выражением Акулова (см. текст), $c$ зависимость константы анизотропии $K_{1}$ от температуры для образцов: $1.2-(1)$ и $3.1-(2)$. 


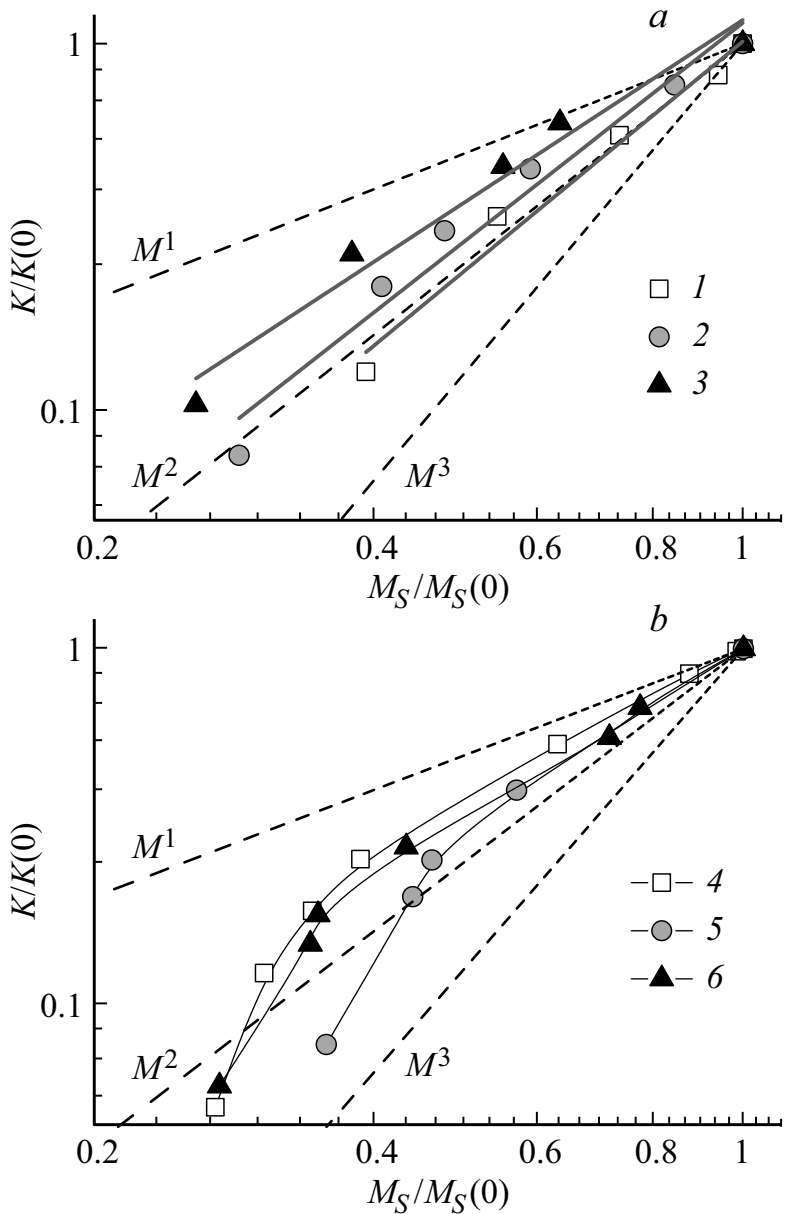

Рис. 2. $a-$ зависимости отношения $K / K(0)$ от $M_{S} / M_{S}(0)$ в двойных логарифмических координатах для образцов: $1.2-(1), 1.3-(2)$ и $2.1-(3)$ (сплошными серыми линиями показана аппроксимация линейной зависимостью), $b$ - зависимости отношения $K / K(0)$ от $M_{S} / M_{S}(0)$ в двойных логарифмических координатах для образцов: $3.1-(4), 3.2-(5)$ и $3.3-(6)$. Сплошными пунктирными линиями показаны расчетные зависимости $K / K(0)=\left(M_{S} / M_{S}(0)\right)^{m}$ с показателями степени $m=1,2$ и 3 .

гадолиния зависимость $K\left(M_{S}\right)$ не является степенной для обоих использованных способов приготовления образцов (рис. $2, b$ ).

\section{4. Обсуждение}

Магнитокристаллическая анизотропия кристалла обычно определяется одноионной анизотропией атомов, из которых он состоит [4]. В модели Нееля анизотропия приписывается парным взаимодействиям и реализуется внутриатомной спин-орбитальной связью. В случае простых магнитов с единственной магнитной подрешеткой ( $\mathrm{Fe}$ или $\mathrm{Co}$ ) теория Callen-Callen является хорошим приближением. В случае интерметаллических редкоземельных магнитов, таких как $\mathrm{SmCo}_{5}$ и $\mathrm{Nd}_{2} \mathrm{Fe}_{14} \mathrm{~B}$, вклады одноионной анизотропии отдельных редкоземельных атомов зависят от температуры как $1 / T^{2}$ [5]. Однако, поскольку магнитный момент формируется вкладом от разных атомов, кристаллическая магнитная анизотропия реализуется межатомной спин-орбитальной связью, которая нарушает выводы одноионной модели $[13,14,18]$.

Другой причиной отклонения от теории Callen-Callen является зеренная структура магнитов и вклад поверхностной анизотропии зерен. В работе [5] дано теоретическое описание температурного поведения поверхностной магнитной анизотропии для частиц разной формы. Было продемонстрировано, что показатель степени $m$ уменьшается с увеличением вклада поверхностной анизотропии потому, что намагниченность поверхности более резко уменьшается с температурой, чем намагниченность материала в объеме частиц. В результате, показатель $m$ сильно зависит от значения поверхностной анизотропии и всегда ниже, чем показатель в случае объемного материала из-за сильных флуктуаций намагниченности на поверхности.

Согласно [14] теория Callen-Callen справедлива только для случаев, когда $A S / H_{\text {ex }} \ll 1$, где $A-$ энергия одноионной анизотропии, $S-$ спин и $H_{\mathrm{ex}}-$ обменное поле. В сплавах, исследованных в данной работе, зависимость $K(T)$ от $M_{S}(T)$ отклоняется от степенного закона только для трех сплавов, содержащих атомы $\mathrm{Gd}$. Примесь гадолиния, имеющего изотропный тензор одноионной анизотропии (в отличие от ионов Dy и $\operatorname{Pr}$ с симметрией тензора типа „легкая ось“) [22], приводит к изменению температурной зависимости магнитной анизотропии (рис. 1,c). Сравнение серий 1 и 2, в которых главным различием в химическом составе была разная концентрация бора, показывает, что небольшие вариации бора не изменяют степенной зависимости константы анизотропии от намагниченности насыщения, а вариации показателя степени $m$ возникают в результате изменений условий приготовления сплава (прямым деформированием или экструзией). Можно также видеть, что структурные вариации (межзеренные расстояния, размер зерен и др. характеристики, зависящие от условий деформации) не изменяют степенной зависимости магнитной анизотропии от намагниченности, в то время, как примесь Gd существенно изменяет ее.

\section{5. Выводы}

Установлено, что в спеченном магните $(\mathrm{PrDy})(\mathrm{FeCo}) \mathrm{B}$ магнитокристаллическая анизотропия $K$ прямо пропорциональна $\left(M_{S}\right)^{m}$ в диапазоне температур $2-300 \mathrm{~K}$ с показателем степени $m=1.7-2.1$ в зависимости от условий изготовления образца. Причинами отклонения от закона Callen-Callen c $m=3$ являются вовлечение двух подрешеток (редкоземельной и подрешетки переходных металлов) в формирование магнитной анизотропии и вклад поверхностной анизотропии на границе зерен. Для образцов, содержащих $\mathrm{Gd}$, зависимость константы анизотропии $K$ от $M_{S}$ перестает быть степенной. Способ 
приготовления образцов (экструзия и прямая деформация) и температура прессования, а также зависящие от них текстура, межзеренное расстояние и др. влияют лишь на величину показателя степени $m$, в то время, как легирование гадолинием радикально меняет тип функции $K\left(M_{S}\right)$.

\section{Список литературы}

[1] M. Sagawa, S. Hirosawa, K. Tokuhara, H. Yamamoto, S. Fujimura, Y. Tsubokawa, R. Shimizu. J. Appl. Phys. 61, 3559 (1987).

[2] J.F. Herbst. Rev. Mod. Phys. 63, 819 (1991).

[3] K. Kumar. J. Appl. Phys. 63, R13 (1988).

[4] R. Skomski, J.M.D. Coey. Permanent Magnetism. Institute of Physics, Bristol (1999). 404 p.

[5] R. Skomski. J. Appl. Phys. 83, 6724 (1998).

[6] R. Skomski, A. Kashyap, D.J. Sellmyer. IEEE Trans. Magn. 39, 2917 (2003).

[7] J.B. Staunton, S. Ostanin, S.S.A. Razee, B.L. Gyorffy, L. Szunyogh, B. Ginatempo, E. Bruno. Phys. Rev. Lett. 93, 257204 (2004).

[8] O.N. Mryasov, U. Nowak, K. Guslienko, R. Chantrell. Europhys. Lett. 69, 805 (2005).

[9] R. Skomski, O.N. Mryasov, J. Zhou, D.J. Sellmyer. J. Appl. Phys. 99, 08E916 (2006).

[10] N. Kobayashi, K. Hyodo, A. Sakuma. Jpn. J. Appl. Phys. 55, 100306 (2016).

[11] N.H. Hai, N.M. Dempsey, D. Givord. IEEE Trans. Magn. 39, 2914 (2003).

[12] R. Skomski, A. Kashyap, J. Zhou. Scr. Mater. 53, 389 (2005).

[13] Y. Toga, M. Matsumoto, S. Miyashita, H. Akai, S. Doi, T. Miyake, A. Sakuma. Phys. Rev. B 94, 174433 (2016).

[14] D. Miura, R. Sasaki, A. Sakuma. Appl. Phys. Express 8, 113003 (2015)

[15] K.J. Strnat. Proc. IEEE 78, 923 (1990).

[16] R. Skomski, P. Manchanda, P.Kumar, B. Balamurugan, A. Kashyap, D.J. Sellmyer. IEEE Trans. Magn. 49, 3215 (2013).

[17] S.D. Bader. Rev. Mod. Phys. 78, 1 (2006).

[18] B.K. Chatterjee, C.K. Ghosh, K.K. Chattopadhyay. J. Appl. Phys. 116, 153904 (2014).

[19] R. Sasaki, D. Miura, A. Sakuma. Appl. Phys. Express 8, 043004 (2015).

[20] N.S. Akulov. Z. Physik 69, 822 (1931).

[21] H.B. Callen, E. Callen. J. Phys. Chem. Solids 27, 1271 (1966).

[22] R. Skomski, D.J. Sellmyer. J. Rare Earths 27, 675 (2009).

Редактор Т.Н. Василевская 